La revue La revue pour l'histoire du CNRS

POUR L'HISTOIRE DU CNRS

1 | 1999

Le CNRS au temps de Charles de Gaulle

Discours prononcé par Charles de Gaulle

Le 14 février 1959 à la Cité universitaire de Toulouse

(2) OpenEdition

Journals

Édition électronique

URL : https://journals.openedition.org/histoire-cnrs/484

DOI : 10.4000/histoire-cnrs.484

ISSN : 1955-2408

Éditeur

CNRS Éditions

Édition imprimée

Date de publication : 6 novembre 1999

ISBN : 978-2-271-05707-5

ISSN : 1298-9800

Référence électronique

"Discours prononcé par Charles de Gaulle », La revue pour l'histoire du CNRS [En ligne], 1 | 1999, mis en ligne le 06 décembre 2006, consulté le 20 mai 2021. URL : http://journals.openedition.org/histoirecnrs/484; DOI : https://doi.org/10.4000/histoire-cnrs.484

Ce document a été généré automatiquement le 20 mai 2021.

Comité pour l'histoire du CNRS 


\title{
Discours prononcé par Charles de Gaulle
}

\author{
Le 14 février 1959 à la Cité universitaire de Toulouse
}

1 Messieurs les Ministres, Monsieur le Recteur, Messieurs les Doyens, Messieurs les Professeurs, Mesdames, Mesdemoiselles, Messieurs,

2 Je vais d'abord simplement vous dire à quel point j'ai été impressionné par ce qu'il m'a été donné de voir, aujourd'hui, à Toulouse. Quand on a vu à la fois cette conjonction de la recherche fondamentale et de ceux qui s'y adonnent, de la recherche appliquée et de ceux qui la font, de l'Enseignement supérieur et de ceux qui y participent, enfin de tout ce qui est pratiquement accompli à partir de là, on reconnaît une harmonie. Or, l'harmonie, c'est, en soi, saisissant et satisfaisant. Mais on se convainc, aussi, que l'intérêt humain et l'intérêt national sont bien servis dès lors qu'il existe cette conjonction entre les travaux et entre les hommes des deux sortes de recherches ainsi que de leurs applications.

3 J'ai donc vu Sud-Aviation, l'École d'agriculture d'Ondes, l'Institut d'optique électronique, l'Institut de toxicité, le Service de la carte de la végétation et des cultures, le Laboratoire électrotechnique et hydraulique, ici même la Cité universitaire. C'est vous dire que je suis fixé.

4 Au moment où je suis de ma vie, bref, dans mes dernières années, j'ai le sentiment, à l'université de Toulouse, de me trouver sur une plage, au bord d'un océan, celui qui peut vous porter, vous les chercheurs, vous les professeurs, vous les étudiants, vers les rivages de la découverte, afin de gagner, à partir de là, les terres inconnues du progrès. Partout paraît ici la manifestation du mouvement général de notre espèce. L'homme, aux prises avec l'Univers, c'est-à-dire d'abord avec lui-même, l'homme cherche, à sortir de soi, à accéder à ce monde nouveau, où les désirs restent infinis, mais où la nature cesse d'être limitée. Cet homme moderne regarde avec passion et avec admiration ce qui est découvert dans les cerveaux de quelques-uns, ce qui est réalisé dans les laboratoires, et ce qui est ensuite appliqué par les techniques modernes. Mais, en même temps, il est guidé par son démon, car la rivalité des États, la lutte des idéologies, l'ambition de dominer, ou bien l'esprit d'indépendance, érigent, au fur et à mesure, en 
armes de guerre, les moyens nouveaux destinés à améliorer la vie. Éternel combat de l'Archange et de Lucifer.

5 Voilà pourquoi il est indispensable que, concurremment à la formation scientifique et technique, la pensée pure, la philosophie qui l'exprime, les lettres qui la font valoir, les arts qui l'illustrent et aussi la morale qui procède de la conscience et de la raison inspirent et orientent cet immense effort d'évolution. Ce n'est pas à la faculté des sciences de Toulouse, entouré comme je le suis par les maîtres et les étudiants de diverses facultés, que j'ai à démontrer pourquoi l'éveil et le développement de l'esprit, par la connaissance de ce qui est beau et par le culte de ce qui est bon, doivent s'associer à la formation scientifique de nos jours.

6 Eh bien! La France, qui a, dans le domaine de la Recherche, tant de traditions et tant de capacités profondes, qui se trouve en plein essor de rajeunissement, qui doit absolument choisir entre le déclin ou bien l'enthousiasme pour ce qui est moderne, la France, dis-je, accueille cette transformation avec espoir et satisfaction.

7 Mais une pareille construction comporte plusieurs étages. À la base, il faut qu'une large partie de la jeunesse française vienne à l'Enseignement scientifique et que les étudiants travaillent bien. Plus haut ce sont les Maîtres, dont il faut qu'ils soient en nombre suffisant et qu'ils aient les moyens voulus pour accomplir leur grande tâche. Plus haut, encore, les Chercheurs, à qui il faut l'équipement spécial nécessaire à leurs travaux et l'art de ne point cloisonner les pensées et les résultats.

8 Au sommet, enfin, l'État! L'État qui a le devoir d'entretenir dans la nation un climat favorable à la Recherche et à l'Enseignement; l'État, qui, malgré le flot des besoins et le flot des dépenses, a la fonction de doter les laboratoires et de pourvoir l'enseignement. L'État, enfin, qui doit orienter l'ensemble, tout en laissant à chacun des chercheurs sa direction et son autonomie. C'est à l'État qu'il appartient de déterminer, dans le domaine de la Recherche, ce qui est le plus utile à l'intérêt public et d'affecter à ces objectifs-là ce dont il dispose en fait de moyens et en fait d'hommes. Eh bien! La France, qui a beaucoup à faire à cet égard, constate que beaucoup a été fait et que beaucoup se fait.

9 J'ai nommé tout à l'heure les témoignages que m'a offerts Toulouse. J'y pourrais ajouter ce qui a été récemment accompli du côté des pouvoirs publics pour organiser au mieux ou créer ce qui devait l'être. Le Conseil supérieur de la recherche scientifique a vu sa tâche précisée. Ont été institués: le Délégué général à la recherche scientifique et technique, le Comité consultatif de la recherche, formé de savants et de chercheurs, et le Comité interministériel qui appelle les ministres et les savants à collaborer directement.

10 Quoi que l'on fasse cependant, tout peut dépendre, tout à coup, de l'éclair imprévu et imprévisible qui jaillit parfois d'un cerveau. Il n'y a aucune raison pour que la France n'enfante pas demain, comme elle l'a fait hier, de ces hommes exceptionnels. Peut-être est-il parmi vous celui-là qui est appelé à accomplir une découverte merveilleuse. Celuilà, je le salue d'avance, et je salue sa gloire future. Mais aussi, par-delà l'université de Toulouse, je salue l'Enseignement français, les chercheurs, les maîtres, les étudiants. En même temps, je leur rends témoignage parce qu'ils servent celui qu'il faut servir, c'està-dire l'homme, tout simplement.

11 Oui ! Rien n'est meilleur que d'alléger le fardeau des hommes. Rien n'est plus noble et plus grand que de lui offrir de l'espoir. 